\title{
Admission Control Methods in IP Networks
}

\author{
Erik Chromy, Marcel Jadron, and Tomas Behul
}

Faculty of Electrical Engineering and Information Technology, Institute of Telecommunications, Slovak University of Technology Bratislava, Ilkovičova 3, 81219 Bratislava, Slovakia

Correspondence should be addressed to Erik Chromy; chromy@ut.fei.stuba.sk

Received 29 January 2013; Accepted 1 March 2013

Academic Editor: Athanasios Vasilakos

Copyright (C) 2013 Erik Chromy et al. This is an open access article distributed under the Creative Commons Attribution License, which permits unrestricted use, distribution, and reproduction in any medium, provided the original work is properly cited.

\begin{abstract}
Telecommunications operators and research institutions pay close attention to the issue of quality of service. The paper deals with methods of admission control in IP networks, which is only one of the subsets of quality of service. There are two large groups of AC methods: parameter-based admission control and measurement-based admission control. The core of the paper is simulation of AC methods and algorithms for topology model in MATLAB. Our simulations are mainly focused on required bandwidth and loss rates. At the end of the paper there are compared results of simulations.
\end{abstract}

\section{Introduction}

Nowadays we face the unstoppable development of information technology, which penetrate into various spheres of users activities and services. Technology make available to end users more and more information. One of the technological advances to which the term information technology is related is a computer network. It is used in many ways, but especially for the transmission of voice, video, and data (i.e., communication). Network provides conditions for Quality of Service (QoS), which lead to the satisfaction of the end user. The process, which significantly affects the QoS, is admission control methods $[1,2]$. The present and future networks are a precondition for the satisfaction of user availability of needed bandwidth. In places where the replacement of infrastructure would be costly it is necessary to develop means for more efficient use of available bandwidth.

One of the tools used in IP Multimedia Subsystem (IMS) technology is an element of Resource Admission Control Subsystem (RACS). RACS is an important logical network element that is used to communicate between the control layer and transmission management functions resource reservation, admission control, support for border gateway services, network address translation, networks aggregation, and QoS support.

Between RACF (Resource Admission Control Function) element and RACS element there are some differences consisting in bandwidth reservation, sharing service addresses across the network. It could be said that RACS is in some way specification of RACF. It was designed by TISPAN organization. A network element is responsible for the implementation of procedures and mechanisms handling policy-based resource reservation and admission control for both unicast and multicast traffics in access networks, core networks, and customer premises networks [3].

RACS reserves appropriate resources and allow the requirement assuming policies, and required resources in the transport network are available. RACS provides the means for operators to promote admission control and option to set policies for service providers. In providing QoS influencing factor is admission control, providing to permit or to denial connection with allocating the required bandwidth. Element of RACS is responsible for admission control, while for defining its architecture is necessary to determine the possible QoS management functions in fixed networks. To ensure the QoS in IMS network, the dynamic QoS management is used. These determine the parameters necessary for operation.

Two classes of management assign parameters by different mechanisms. Guaranteed control uses a mechanism to set limits for some or all of the QoS parameters such as delay, variable delay, throughput, and packet loss. This is achieved by admission control itself and by decisions of admission control in access network by management of throughput and traffic policies. Relative management distinguished class service by applying the appropriate QoS mechanism taking into consideration the user's device capabilities. 
The rest of this paper is organized as follows. Section 2 is devoted to the description of the two basic groups of AC methods: parameter based admission control methods (PBAC) and measurement based admission control methods (MBAC). In Section 3 there are simulated PBAC and MBAC methods and MBAC algorithms. Section 4 summarizes the results of simulations.

\section{Admission Control Methods}

Using the admission control methods is possible to propose an access control model, which will provide QoS [4-10]. Created models can be used separately, or they can complement each other (in order to achieve better QoS). To ensure that the quality is the most important step control access of incoming data flows. This control is provided by AC method. The main purpose of these methods is to estimate the expected bandwidth for the incoming data stream and determine whether it is possible to assign needed bandwidth and effectively use of resources, to avoid congestion. AC methods are most used for delay-sensitive and jitter-sensitive services, respectively, for real-time applications. There are a lot of admission control methods, and their difference consists mainly in the different types of operation and method of implementation. Some AC methods are based on mathematical calculations and statistical indicators, others on measuring traffic.

In general, the control methods are represented by two categories: parameter based admission control (PBAC) and measurement based admission control (MBAC). PBAC method is based only on the current active traffic and on the characteristics of the total active traffic. This method may not be optimal, because it does not rely on a new incoming traffic. MBAC method does not specify for obtaining the parameters of the source, but to make measurements on the network running in real time. This method achieves higher network utilization because serving a new data flows in network is common. Common criteria of admission control methods are the ability to allocate bandwidth for all actual data flows in case of not exceeding the total capacity of the line. It is very important that the nodes on which the method is applied to are in direct contact with QoS parameters (e.g., delay, loss rates). The result is QoS produced by algorithm, which should be independent of traffic type and should be as close to the desired value as it is possible. In this case it is not necessary that an administrator has to change the AC algorithm value in case of changing the traffic type [11].

2.1. Parameter-Based Admission Control. PBAC may be preferred because of their ease of implementation in network management. They work with parameters such as peak or the effective bandwidth of incoming data flow instead of the values measured in the network. Using PBAC methods is possible to avoid the limitations incurred by measuring and monitoring the network. In these methods, there may be unequal use of network resources when some data flows are often idle or sending at the top transmission rates. In this case, the new data stream may be rejected even though it would not affect the QoS of ongoing data flows [12].
2.2. Measurement-Based Admission Control. MBAC methods use measurement of the current state of network traffic, if present, to support the incoming data flow. MBAC methods perform decision-making process, which depends on the measurement of the traffic and QoS parameters. In case of measuring and monitoring the network, we can more effectively use resources (compared to PBAC [13]). MBAC methods offer the possibility of providing QoS to priori data flows. When compared to the PBAC methods, MBAC methods are adaptive, since measurement is performed at specific intervals, and so there is no waste of resources [14].

\section{Simulation}

The following chapter deals with simulation of AC methods using topology in Figure 1.

Admission control method is applied to the router. A precondition of the displayed topology is a number of users who generate random data flow at the time. These requirements represent random rates of data, from sources, so none of the sources does not have a constant bit rate. Each source needs different requirements of bandwidth. Speeds range from 0 to $128 \mathrm{kbit} / \mathrm{s}$. When simulating a network with a large number of users, it is appropriate to use a Gaussian distribution [14].

3.1. PBAC Methods. The main feature of PBAC is decision making, which does not consider the possibility of a new data flow, resulting from (1). The value of the probability of packet loss is $\varepsilon=10-3$, corresponding to the network [15]. The first method is simulated in PBAC. Simulation is based on (1). A precondition of simulation is 5000 users, spawning requirements for allocating bandwidth in 500 different times as follows:

$$
c=m+a^{\prime} \sigma
$$

where

$$
a^{\prime}=\sqrt{-2 \ln (\varepsilon)-\ln (2 \pi)}
$$

where, $c$ is bandwidth [bit/s], $\sigma$ is standard variation of aggregate bit rate [bit/s], $\varepsilon$ is upper limit of the probability of overflow, and $m$ is average bit rate of aggregate traffic [bit/s].

In Figure 2 it is evident that with the growing number of users in the network, bandwidth requirements grow. When using more resources, allocated bandwidth value would acquire larger values. The comparison shows the difference between values generated using Gaussian distribution and random distribution in MATLAB environment. In case of Gaussian distribution it is necessary to consider the greater allocated bandwidth.

The following simulations are because of higher number of resources used Gaussian distribution, as it looks more real than random distribution offered by the program MATLAB. In the next simulation (Figure 3) it is evident that in case of using PBAC methods it is necessary to provide higher bandwidth, but without the application of admission control method that could be used for transmission line with 


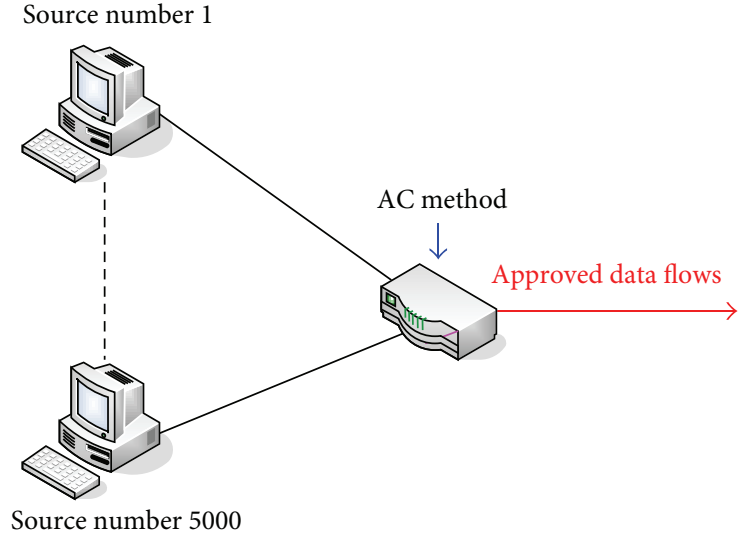

FIGURE 1: Simulated topology.

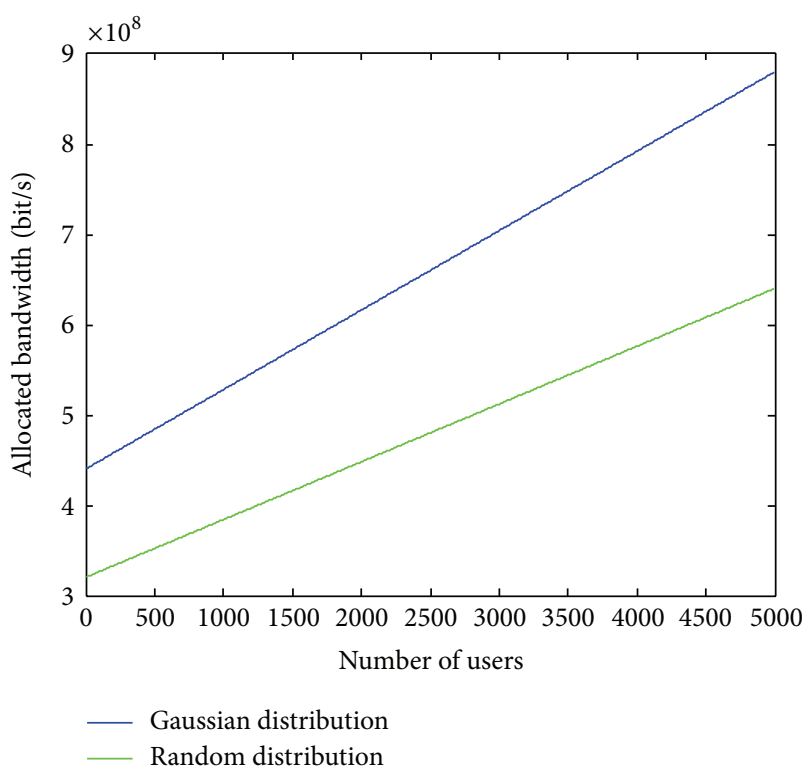

FIGURE 2: Simulation of allocating required bandwidth.

less transmission capacity. But that fact would constitute deficiency in providing QoS in the network. Admission control puts certain demands on the network, but these are a guarantee of quality. The difference of allocated bandwidth, respectively, difference between network with applied method and network without the application of any method is shown in Figure 3. The process describes a constant value, as is clear from (1) and (2).

3.2. MBAC Methods. Another simulated method is measurement based admission control (MBAC). This method is characterized by (2) and (3) as follows:

$$
c_{\text {est }}=m_{\text {measure }}+p_{\text {new }}+a^{\prime} \sqrt{\sigma_{\text {measure }}^{2}}
$$

where, $c_{\text {est }}$ is estimated bandwidth $[\mathrm{kbit} / \mathrm{s}], m_{\text {measure }}$ is average bit rate of source in time moment $[\mathrm{kbit} / \mathrm{s}], p_{\text {new }}$ is bit rate of new data flow $[\mathrm{kbit} / \mathrm{s}]$, and $\sigma^{2}$ measure is variance.

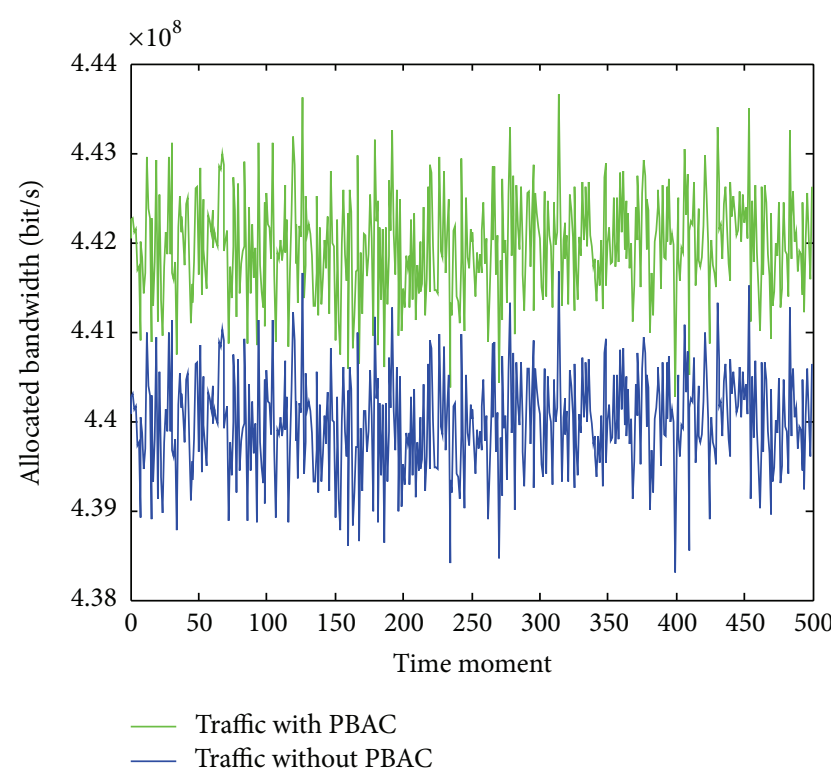

FIGURE 3: Simulation of allocated bandwidth of the applied method PBAC and without applied PBAC methods.

Typical feature of this method is measurement which is done only for a certain range of samples, which reduce requirements on memory. Simulations are used all the resources, but each of them uses 150 samples representing different requirements for bandwidth at different times (the simulation are used time samples from 50 to 199). It is obvious, that with increasing requirements on communication medium, the required bandwidth grows. Simulated method could achieve the best results in view of accuracy of the allocated bandwidth to ensure QoS if used all the time samples (i.e., 500 samples). In the case of large-scale samples as input data, there would be time-consuming calculations which could lead to increase of delay and jitter, which is not suitable for real-time applications. It also would plac higher demands on memory. In case of using PBAC method that does not considered new possible data flow are allocated smaller bit rates, but works with all time samples. In MBAC method it is larger allocated bandwidth, because this method considered a new possible data flow and value of statistical variation for both simulated methods vary from each other. In Figure 4, it is obvious that the ensuring of QoS is better for MBAC methods than PBAC methods in case of new incoming data flow.

From the simulation it is also clear that the increased number of samples at a time leads to higher allocation of bandwidth. The optimal number of samples is selected based on the workload on the network. For comparison, if the method worked with a number of 50 sample, the value of the allocated bandwidth would be smaller than the number of samples at 400 . The higher number of samples requires more memory to work with them, causing higher levels of delays. Based on the estimated bandwidth and capacity of output link $C$, the method decides to accept or reject the connection. In decision takes an important function factor APF 


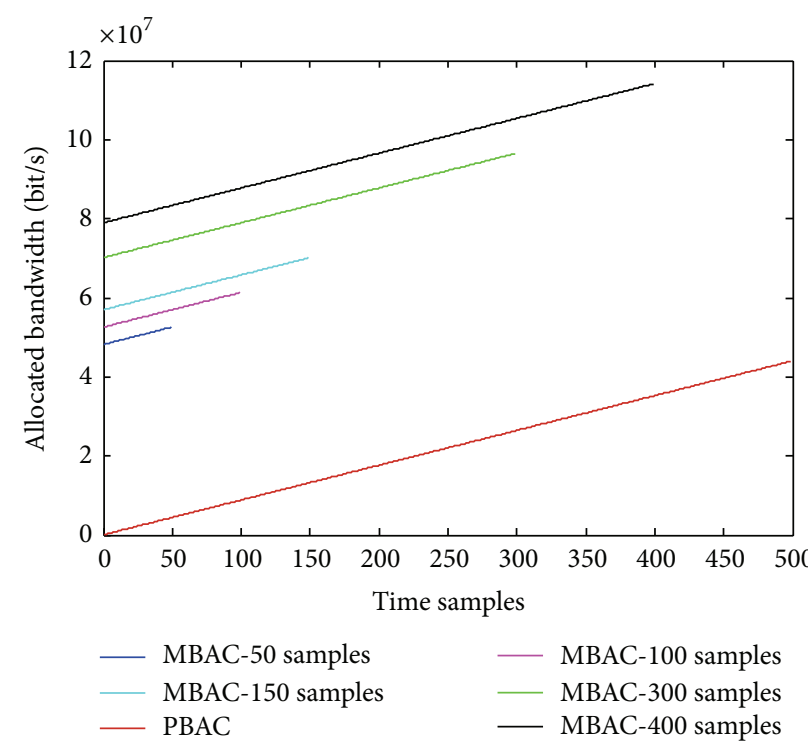

FIGURE 4: Increase of allocated bandwidth.

(Admission Policy Factor-factor decision), which regulates the inaccuracy of the method for different data flows and their clusters [16]. APF also indicates what is owed on bandwidth as follows:

$$
\begin{aligned}
& \text { if } c \geq\left(c_{\mathrm{est}} * \mathrm{APF}\right), \text { to accept a new flow, } \\
& \text { if } c \leq\left(c_{\mathrm{est}} * \mathrm{APF}\right), \text { to reject a new flow, }
\end{aligned}
$$

where, $c_{\text {est }}$ is an estimated bandwidth using the method [bit/s], and $C$ is link capacity [bit/s].

When requesting bandwidth unsatisfactory inequalities (4) data flow would be rejected, which would have an impact on QoS parameters such as delay, jitter, and loss rates.

In comparison of simulation (in Figure 5) it is clear that in the network with admission control there is allocated larger bandwidth. Simulation is applied to a given sample, respectively, 150-time moments in which sources placed certain requirements for the allocation of bandwidth. Size variations of these operations is shown in Figure 6.

The Figure 7. shows the characteristics of the estimated bandwidth for different numbers of samples in network with applied MBAC method and without MBAC. It is obvious, that by use of 400 samples it is allocated larger bandwidth than by use of 50 samples, which can be observed also in Figure 4. Thus the value of samples is necessary to change, based on network utilization for optimal functioning of the method.

3.3. MBAC Algorithms. MBAC methods relate to MBAC algorithms that are used to estimate the bandwidth of data flows [17]. The algorithm MBACs determines the effective bandwidth for the aggregate traffic by

$$
C(s)=\frac{1}{s} \sum_{k=1}^{N} \ln \left(1+\frac{e^{s h_{k}}-1}{h_{k}} * m_{k}\right)+\frac{\gamma}{s},
$$

where, $C(s)$ is estimated bandwidth for the aggregate traffic $[\mathrm{kbit} / \mathrm{s}], h_{k}$ is peak rate of source $[\mathrm{kbit} / \mathrm{s}], m_{k}$ is average

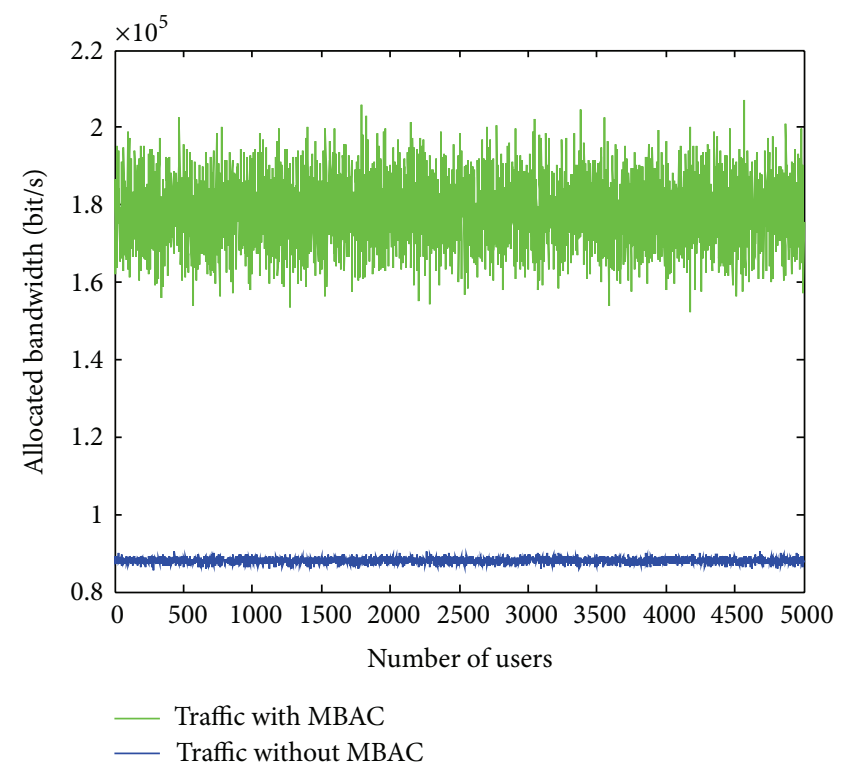

FIGURE 5: Comparison of traffic with applied MBAC method and without MBAC methods.

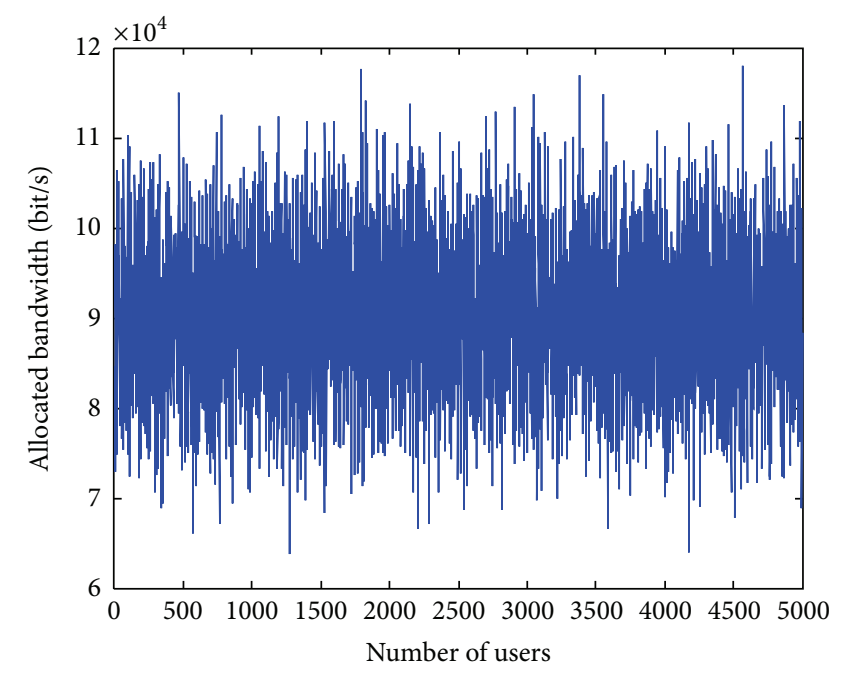

FIGURE 6: Variation with MBAC method.

source bit rate $[\mathrm{kbit} / \mathrm{s}], s$ is compromise between the use of transmission capacity and packet loss (6), and $N$ is number of data flows as follows:

$$
\begin{aligned}
& s= \\
& \sqrt{\frac{\gamma}{(1 / 8) \sum_{k=1}^{N} h_{k}^{2}-(1 / 2 * N) *\left[\sum_{k=1}^{N} m_{k}-(1 / 2) * \sum_{k=1}^{N} h_{k}\right]^{2}}} \\
& \gamma=-\ln (\varepsilon) .
\end{aligned}
$$

The characteristic of this method is the measurement process which is performed on each data flow, causing delays. Improvement of the algorithm is an algorithm MBACf 


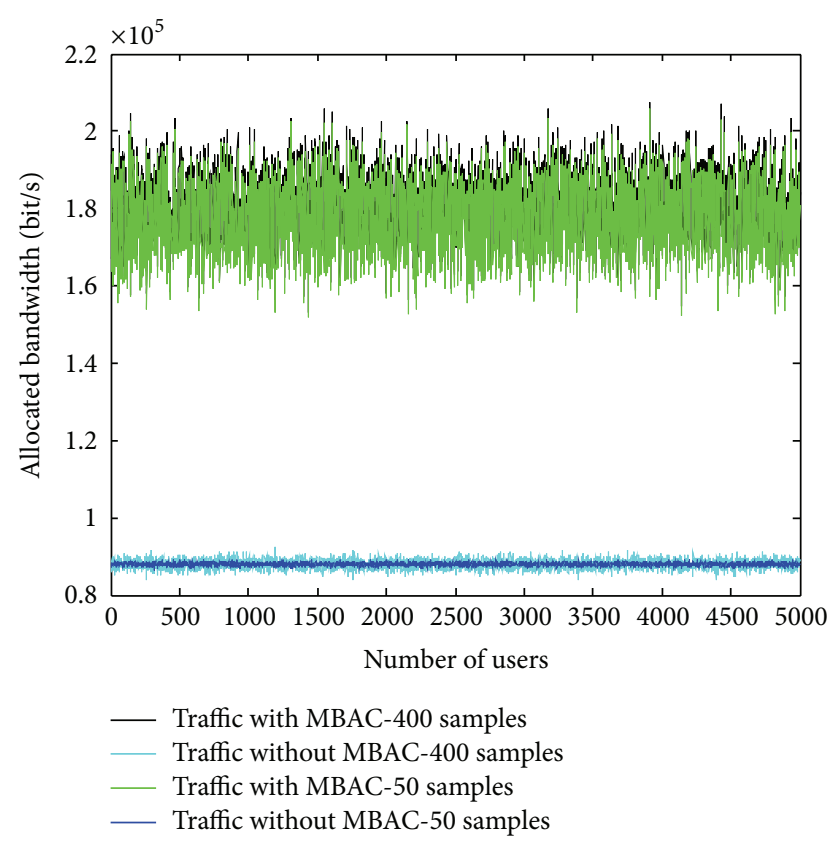

FIGURE 7: Allocating of bandwidth based on the number of samples.

which considers average aggregate operation, which facilitates calculations. Estimated bandwidth at this algorithm is determined by (8). A parameter $s$ is determined by (6) as follows

$$
\begin{aligned}
& C f_{\text {opt }}(s)=\frac{1}{s} \sum_{k=1}^{N}\left[\frac{\sum_{j=1}^{N} m_{j}+\sum_{j=1}^{N}\left(h_{j} /\left(e^{s h_{j}}-1\right)\right)}{N}\right.\left.* \frac{e^{s h_{k}}-1}{h_{k}}\right] \\
&+\frac{\gamma}{s} .
\end{aligned}
$$

In determining the effective bandwidth in MBACs and MBACf methods are used measured average values and peak values of data flows. These methods can be improved by adding a parameter measured variance. The more information is available about the flow, and the more accurate bandwidth can be allocated to him [18]. Using the variance to determine the bandwidth algorithm MBACv works using

$$
\begin{aligned}
& C f_{\text {var }}(s) \\
& =\frac{1}{s} \sum_{k=1}^{N}\left[\frac{\sum_{j=1}^{N} \sigma_{j}^{2}+\sum_{j=1}^{N}\left(h_{j}^{2} /\left(e^{s h_{j}}-1-s h_{j}\right)\right)}{N}\right. \\
& \left.\quad * \frac{e^{s h_{k}}-1-s h_{k}}{h_{k}^{2}}\right] \\
& +\frac{\gamma}{s}+\sum_{k=1}^{N} m_{k},
\end{aligned}
$$

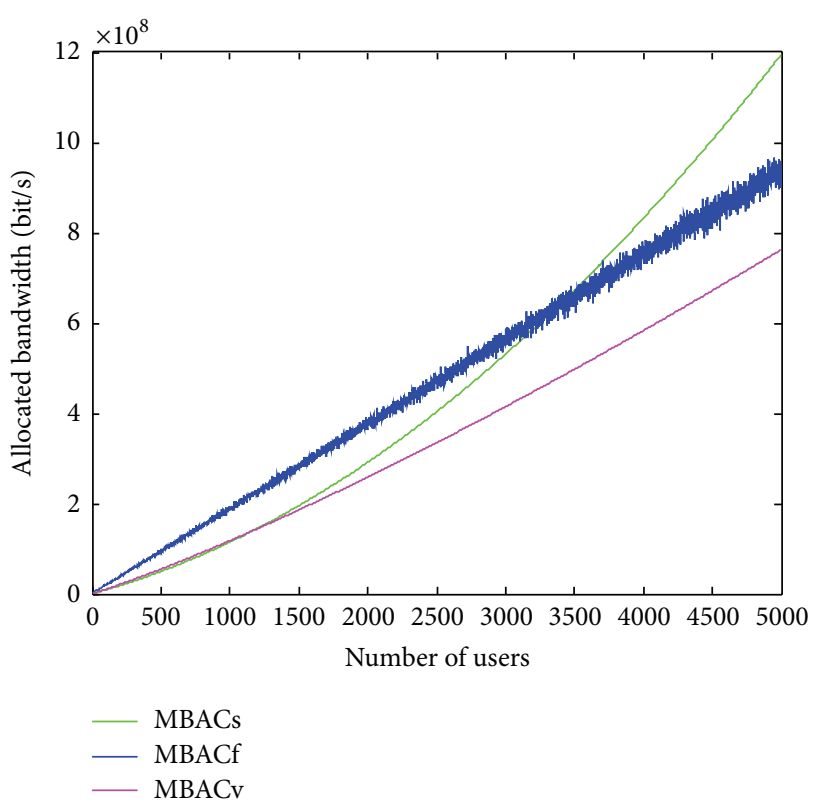

FIGURE 8: Allocating of bandwidth for the growing number of users.

where $\sigma$ is variance,

$s=$

$\sqrt{\frac{\gamma}{(1 / 2) * \sum_{k=1}^{N} \sigma^{2}{ }_{k}+(1 / 18) * \sum_{k=1}^{N} h_{k}^{2}-(1 / 18) *(1 / N) *\left[\sum_{k=1}^{N} h_{k}\right]^{2}}}$.

From characteristics shown in Figure 8, it is clear that using an algorithm for allocation of efficient bandwidth, MBACs algorithm allocates smaller bandwidth than the other two algorithms. However, this trend takes place only in the simulated network using 5000 users, only after accumulating of first 2100 users. Thus, the algorithm is able to some extent to accept more data flows than algorithms MBACf and MBACv. With this algorithm it is therefore possible in the network in which at one moment not all of $100 \%$ users transmit to allocate less bandwidth. When accessing a large number of users, the amount of allocated bandwidth does not change linearly but increasing. At high network capacity utilization levels of allocated bandwidth are higher, thus previous comparison takes the opposite trend, respectively method MBACs serve smaller number of users. Compromise of algorithms MBACs and MBACf is MBACv algorithm, which on whole range of users allocates the necessary effective bandwidth almost linearly with wasted bandwidth at least. The simulation is used in network loss rates $\varepsilon=10-3$, which corresponds to the network. Acceptable amount of loss in the network is $5 \%[19,20]$. In the simulation shown in Figure 9, comparison is shown for allocating bandwidth in networks with different loss rates. In a network with a smaller loss rates, the value allocated bandwidth is higher because reduction in the loss rate parameter increases $\gamma$ and thus changes the parameter $s$. 


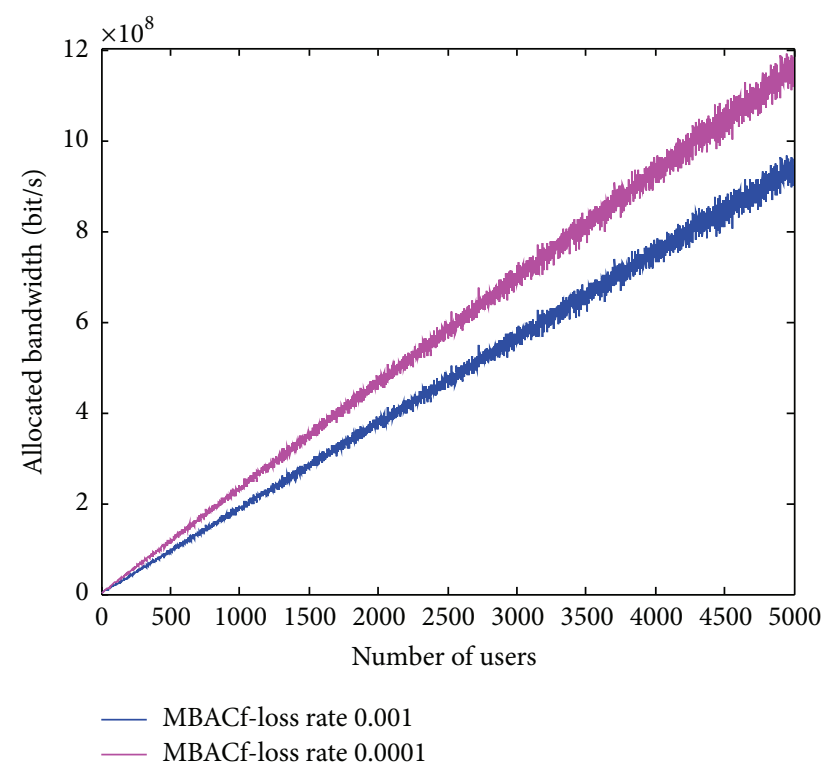

FIGURE 9: Allocating of bandwidth for the growing number of users for different loss rates in the network.

\section{Conclusion}

The paper describes the methods of admission control, used to allocate bandwidth for data flows, respectively, their acceptance or rejection. Different methods and algorithms are used different variables, due to accurate measurement and description of resources and for allocation the best possible bandwidth. The purpose of simulation network topology has been designed with the necessary traffic.

Simulations of MBAC methods are also very important to select the number of samples that enter the simulation. From the simulations it is clear that the increase the number of samples, which operates MBAC method, needs more memory and also increases the time needed to decide on acceptance, respectively, rejecting the new flow. The increasing number of samples entering the simulation increases the estimate of the required bandwidth.

The paper was simulated three different MBAC algorithms MBACs, MBACf, and MBACv. Based on realized simulations, it can be argued that the algorithm MBACv is suitable from the point of view equitable allocation of bandwidth. Finally, simulations have shown parameter loss rates that have a significant impact on the required bandwidth.

In future work, we will focus on the determination of MBAC algorithm, which is suitable for a certain type of traffic: voice over IP [21-24] and IPTV [25]. Each type of traffic, has their own specific characteristics (peak cell rate, average cell rate, burst, etc.) to be considered when assessing the quality and thus has a significant impact on the selection of suitable MBAC algorithm.

\section{Acknowledgments}

This work is a part of research activities conducted at Slovak University of Technology Bratislava, Faculty of Electrical Engineering and Information Technology, Institute of Telecommunications, within the scope of the projects "Support of Center of Excellence for SMART Technologies, Systems and Services II, ITMS 26240120029, cofunded by the ERDF" and project VEGA no. 1/0106/11 "Analysis and Proposal for Advanced Optical Access Networks in the NGN Converged Infrastructure Utilizing Fixed Transmission Media for Supporting Multimedia Services."

\section{References}

[1] A. F. Atlasis, N. H. Loukas, and A. V. Vasilakos, "Use of learning algorithms in ATM networks call admission control problem: a methodology," Computer Networks, vol. 34, no. 3, pp. 341-353, 2000.

[2] M. Kavacký and I. Baroňák, "Evaluation of two statistical CAC methods for variable bit rate traffic sources," Journal of Electrical Engineering, vol. 59, no. 4, pp. 178-186, 2008.

[3] ETSI ES 282001 v3. 4. 1: Telecommunications and Internet converged Services and Protocols for Advanced Networking (TISPAN), NGN Functional Architecture. September 2009.

[4] T. Balogh and M. Medvecky, "Average bandwidth allocation model of WFQ," Modelling and Simulation in Engineering, vol. 2012, Article ID 301012, 7 pages, 2012.

[5] M. Halas and S. Klucik, "Modelling the probability density function of IPTV traffic packet delay variation," Journal Advances in Electrical and Electronic Engineering, vol. 10, no. 4, pp. 225-263, 2012.

[6] T. Misuth and I. Baronak, "Application of M/G/1/K model for aggregated VoIP traffic packet loss estimation," in Proceedings of the 35th International Conference on Telecommunication and Signal Processing (TSP’12), pp. 42-46, Prague Czech Republic, 2012.

[7] R. Róka and F. Čertík, "Modeling of environmental influences at the signal transmission in the optical transmission medium," International Journal of Electrical Communication Networks and Information Security, vol. 4, no. 3, pp. 144-162, 2012.

[8] R. Róka, "The designing of NG-PON networks using the HPON network configurator," Journal of Communication and Computer, vol. 9, no. 6, pp. 669-678, 2012.

[9] A. Vasilakos, C. Ricudis, K. Anagnostakis, W. Pedrycz, and A. Pitsillides, "Evolutionary-fuzzy prediction for strategic QoS routing in broadband networks," in Proceedings of the 1998 IEEE International Conference on Fuzzy Systems, pp. 1488-1493, May 1998.

[10] J. Rozhon and M. Voznak, "Development of a speech quality monitoring tool based on ITU-T P. 862," in Proceedings of the 34th International Conference on Telecommunications and Signal Processing (TSP' 11), pp. 62-66, Budapest, Hungary, August 2011.

[11] M. Grossglauser and D. Tse, "Framework for robus measurement-based admission control," in Proceedings of the Special Interest Group on Data Communication SIGCOMM is the flagship annual conference of the Special Interest Group on Data Communication (SIGCOMM '97), Cannes, France, 1997.

[12] S. Yerima, "Implementation and evaluation of measurementbased admission control schemes within a converged networks QoS management framework," International Journal of Computer Networks \& Communications, vol. 3, no. 4, pp. 137-152, 2011.

[13] E. Chromy, T. Behul, and M. Kovacik, "Admission control methods in IP networks," in Proceedings of the 14th International 
Conference on Research in Telecommunication Technologies (ICACT '12), pp. 93-97, Bratislava, Slovakia, September 2012.

[14] F. Brichet and A. Simonian, "Conservative gaussian models applied to measurement-based admission control," in Proceedings of the 6th International Workshop on Quality of Service (IWQoS '98), pp. 68-71, San Francisco, Calif, USA.

[15] J. Micuch and I. Baronak, "Admission control methods for ensuring quality of service in IP," Posterus, vol. 3, no. 5.

[16] E. Alipour and K. Mohammadi, "Adaptive admission control for QoS guarantee in differentiated services networks," International Journal of Computer Science and Network Security, vol. 8, no. 6, pp. 93-98, 2008.

[17] K. Frank, S. Zachary, and I. Ziedins, "Notes on effective bandwidths," in Stochastic Networks: Theory and Applications, Royal Statistical Society Lecture Notes Series, pp. 141-168, Oxford University Press, New York, NY, USA, 1996.

[18] Z. Turányi, A. Veres, and A. Oláh, A Family of MeasurementBased Admission Control Algorithms. Trafic Analysis and Network Performance Laboratory, Chapman \& Hall, Budapest, Hungary, 1996.

[19] R. J. Gibbens, F. P. Kelly, and P. B. Key, "Decision-theoretic approach to call admission control in ATM networks," IEEE Journal on Selected Areas in Communications, vol. 13, no. 6, pp. 1101-1113, 1995.

[20] G. Almes, S. Kalidindi, and M. Zekauskas, "A one-way packet loss metric for IPPM," RFC 2680, 1999.

[21] M. Voznak and F. Rezac, "Threats to voice over IP communications systems," WSEAS Transactions on Computers, vol. 9, no. 11, pp. 1348-1358, 2010.

[22] M. Voznak and J. Rozhon, "Methodology for SIP infrastructure performance testing," WSEAS Transactions on Computers, vol. 9, no. 9, pp. 1012-1021, 2010.

[23] M. Voznak and F. Rezac, "Web-based IP telephony penetration system evaluating level of protection from attacks and threats," WSEAS Transactions on Communications, vol. 10, no. 2, pp. 6676, 2011.

[24] B. Kyrbashov, I. Baroňák, M. Kováčik, and V. Janata, "Evaluation and investigation of the delay in voip networks," Radioengineering, vol. 20, no. 2, pp. 540-547, 2011.

[25] I. Baronak and L. Kočkovič, "Alternatives of providing IPTV using IMS," International Journal of Computers \& Technology, vol. 3, no. 2, pp. 188-192, 2012. 

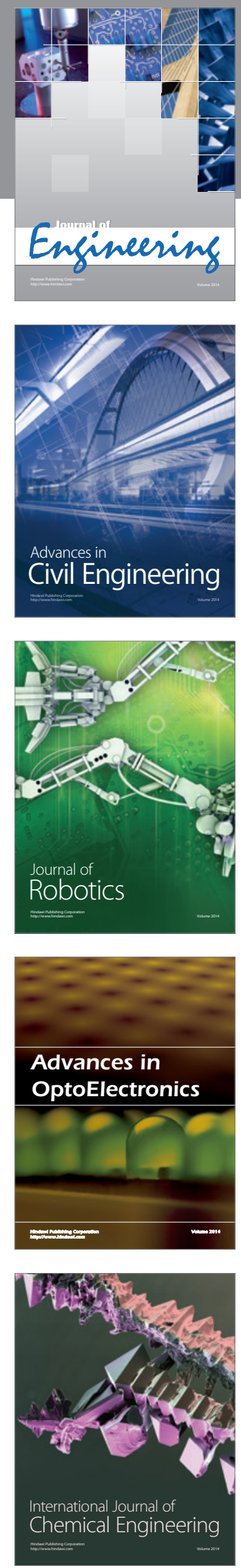

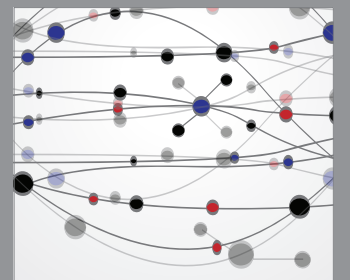

The Scientific World Journal
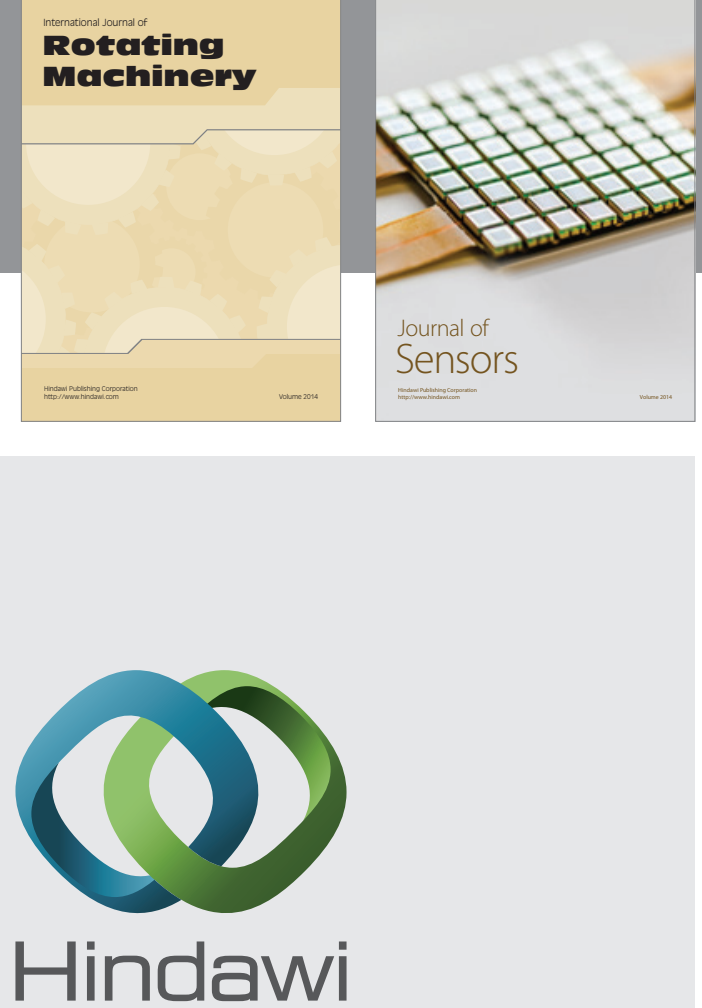

Submit your manuscripts at http://www.hindawi.com
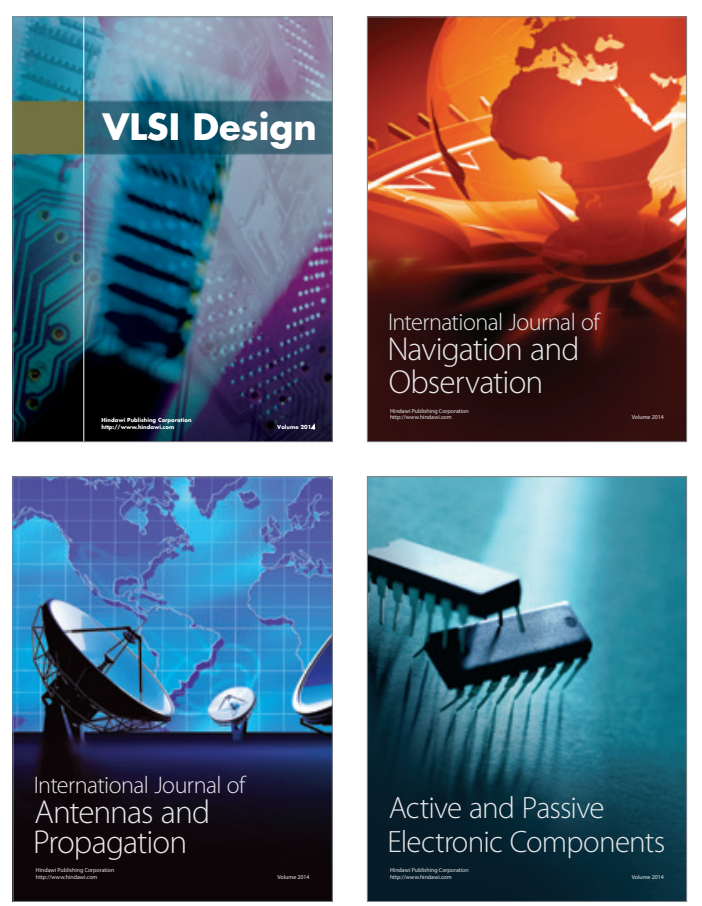
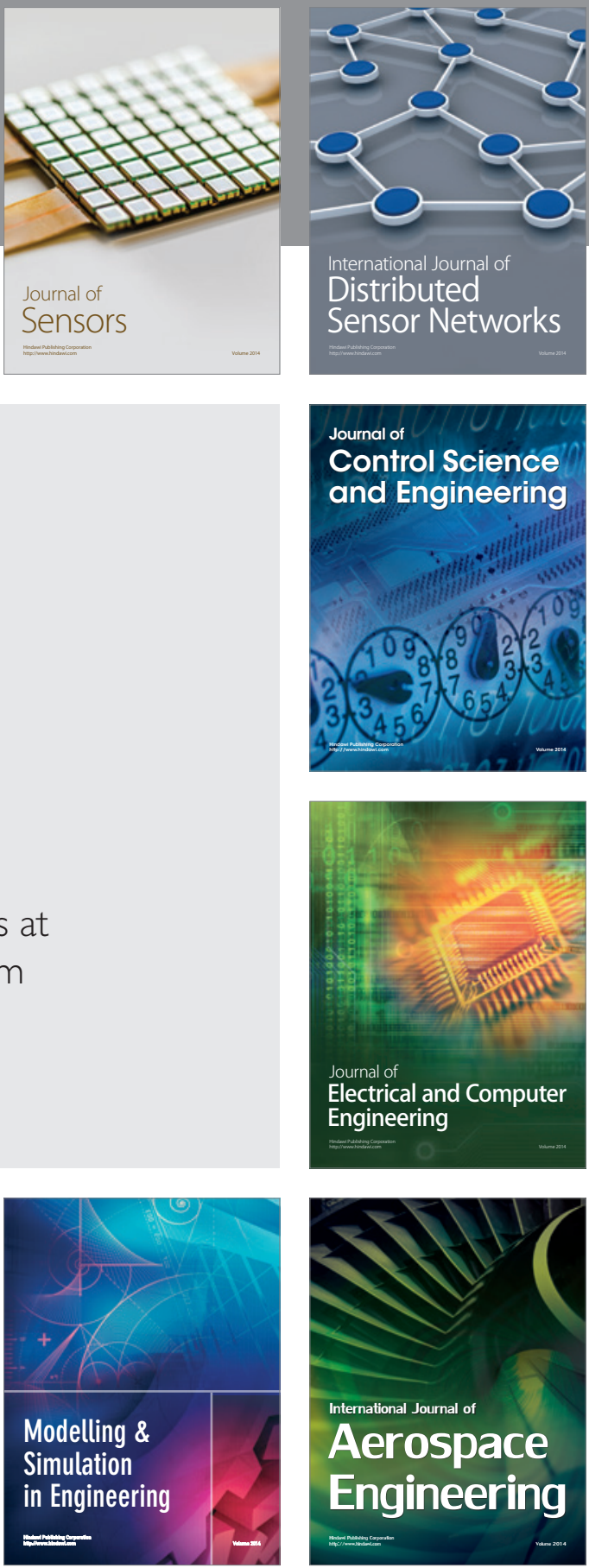

Journal of

Control Science

and Engineering
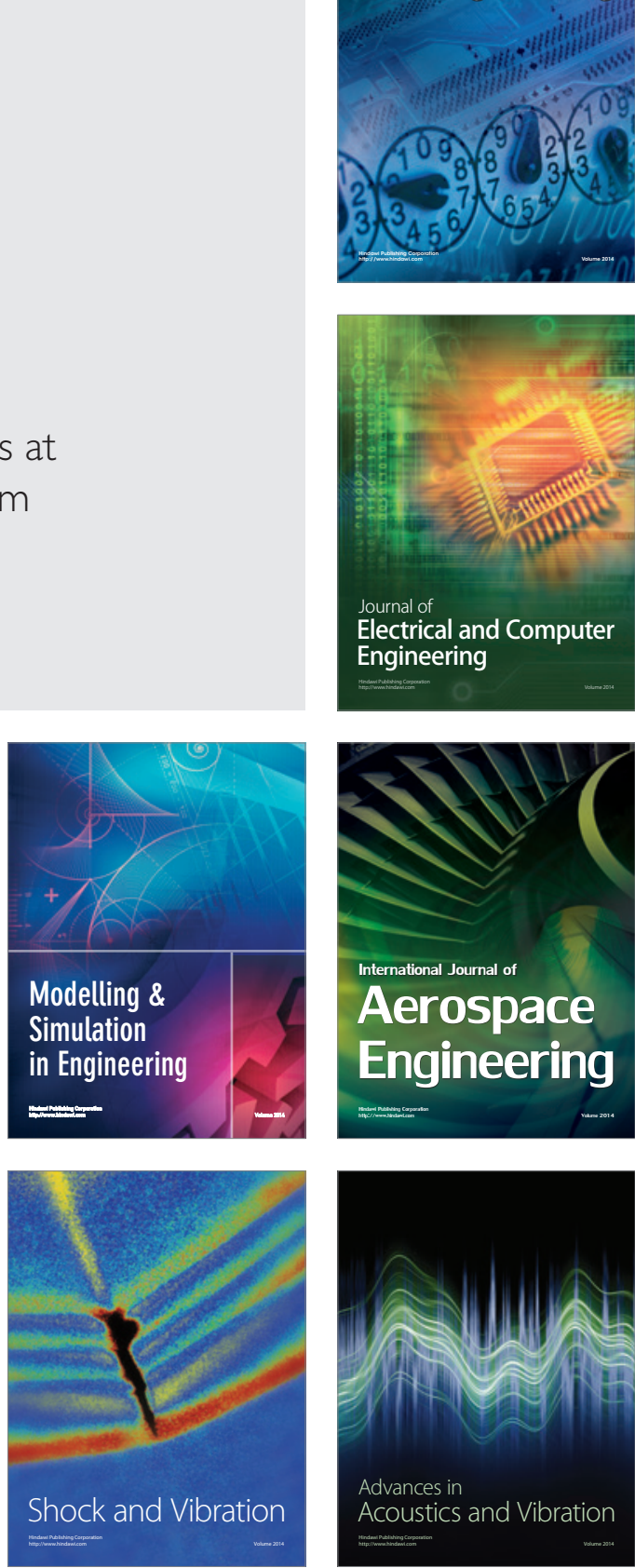